\title{
MEMS-based contact stress field measurements at a rough elastomeric layer: local test of Amontons' friction law in static and steady sliding regimes
}

\author{
J. Scheibert ${ }^{12, a}$, E. Katzav ${ }^{13}$, M. Adda-Bedia ${ }^{1}$, J. Frelat ${ }^{4}$, A. Prevost ${ }^{1}$, and G. Debrégeas ${ }^{1}$ \\ 1 Laboratoire de Physique Statistique, CNRS/ENS/University Paris 6/University Paris 7, Paris, France \\ 2 Physics of Geological Processes, University of Oslo, Oslo, Norway \\ 3 Department of Mathematics, King's College, London, United Kingdom \\ 4 IJLRDA, CNRS/Université Paris 6, Paris, France
}

\begin{abstract}
We present the results of recent friction experiments in which a MEMS-based sensing device is used to measure both the normal and tangential stress fields at the base of a rough elastomer film in frictional contact with smooth, rigid, glass indentors. We consider successively multicontacts under (i) static normal loading by a spherical indentor and (ii) frictional steady sliding conditions against a cylindrical indentor, for an increasing normal load. In both cases, the measured fields are compared to elastic calculations assuming (i) a smooth interface and (ii) Amontons' friction law. In the static case, significant deviations are observed which decrease with increasing load and which vanish when a lubricant is used. In the steady sliding case, Amontons' law reproduces rather satisfactorily the experiments provided that the normal/tangential coupling at the contact interface is taken into account. We discuss the origin of the difference between the Amontons fields and the measured ones, in particular the effect of the finite normal and tangential compliances of the multicontact interface.
\end{abstract}

\section{Introduction}

Knowledge of the surface and subsurface stress fields at the contact region between two solids is of considerable interest to numerous domains such as mechanical engineering (e.g. [1-5]), solid friction [6-12], biomechanics (e.g. [13-17]) or seismology (e.g. [18,19]). From a continuum mechanics point of view, the theoretical or numerical determination of these fields require a set of constitutive equations characterizing the mechanical response of the interface, which are usually inferred from macroscopic measurements. Most practical contact situations involve rough solids for which the interface is a collection of isolated load-bearing points forming a multicontact interface (MCI). Macroscopic MCI usually obey the empirical Amontons' friction law which states that irreversible sliding occurs when the ratio of tangential to normal forces reaches a static friction coefficient $\mu_{\text {macro }}$ without any prior deformation of the interface $[7,20]$. The classical approach for calculating contact stress fields for MCIs therefore consists in considering a smooth interface exhibiting an analog rigid-plastic response: $\mu_{\text {macro }}$ now defines the threshold ratio between shear and normal stress components for local slip to occur [21-24]. Only few recent works tried to explicitely take into account the effect of the finite tangential compliance of MCIs on the contact stress [25,26].

Considering both a smooth interface and Amontons' law may seem paradoxical since the latter is expected to be valid only for rough solids. Because of adhesion forces, contact between molecularly smooth surfaces generally do not obey Amontons' friction law [27,28]. Furthermore, random roughness provides a microscopic basis to Amontons' law [29-32]: for standard roughness characteristics,

a e-mail: julien.scheibert@fys.uio.no 
the real area of contact - and thus the tangential force required to trigger sliding - grows linearly with the applied load.

When associated with Amontons' law, the smooth interface hypothesis must therefore be understood as the limiting case of a macroscopic surface bearing vanishingly small roughness. One may wonder to what extent this approximation is valid in real MCI's. Experimentally, this question has been addressed by focusing on the relationship between the macroscopic normal load and the indentation depth[33,34,31,35,36]. Here we go further and compare the stress field measured within an extended MCI with calculations assuming both smooth surfaces and the local (rigid-plastic) Amontons' law. We consider two differents situations: (i) static sphere-on-plane MCIs under purely normal load, modeled using finite elements (section 3) and (ii) steady-sliding cylinder-on-plane MCIs, for which we developed an original semi-analytical calculation (section 4).

\section{Experimental setup}

In order to perform such local measurements, we take advantage of the recent development of Micro Electro Mechanical Systems (MEMS) sensors [37-39]. Local contact stress measurements are performed with a MEMS force sensor embedded at the rigid base of an elastomer film (Fig. 1). The MEMS' sensitive part (Fig. 1, inset) consists of a rigid cylindrical post (diameter $550 \mu \mathrm{m}$, length $475 \mu \mathrm{m}$ ) attached to a suspended circular Silicon membrane (radius $1 \mathrm{~mm}$, thickness $100 \mu \mathrm{m}, 330 \mu \mathrm{m}$ below the MEMS top surface). When a force is applied to the post, the resulting (small) deformations of the membrane are measured via a bridge of four couples of piezo-resistive gauges embedded in it (inset of Fig. 1). The MEMS thus allows to measure simultaneously the applied stress along three orthogonal directions, averaged over the MEMS's millimetric extension, in a way that will be determined through calibration.

In the present experiments, the MEMS sensor is located at the rigid base of a rough, nominally flat elastomer film of uniform thickness $h=2 \mathrm{~mm}$ ( $\sim 4$ times larger than the post's diameter $)$ and lateral dimensions $50 \times 50 \mathrm{~mm}$. The elastomer is a cross-linked Poly(DiMethylSiloxane) (PDMS, Sylgard 184, Dow Corning) of Young's modulus $E=2.2 \pm 0.1 \mathrm{MPa}$ and Poisson's ratio $v=0.5$. The film is obtained by molding against a Poly(MethylMethAcrylate) plate roughened by abrasion, the resulting rms surface roughness being measured with an interferential optical profilometer (M3D, Fogale Nanotech) to be $1.82 \pm 0.10 \mu \mathrm{m}$. When the film is put in contact against an indentor, the normal and tangential loads applied, respectively $P$ and $Q$ are measured through the extension of two orthogonal loading cantilevers (normal stiffness $641 \pm 5 \mathrm{~N} . \mathrm{m}^{-1}$, tangential stiffness $51100 \pm 700 \mathrm{~N} . \mathrm{m}^{-1}$ ) by capacitive position sensors (respectively MCC30 and MCC5, Fogale nanotech).

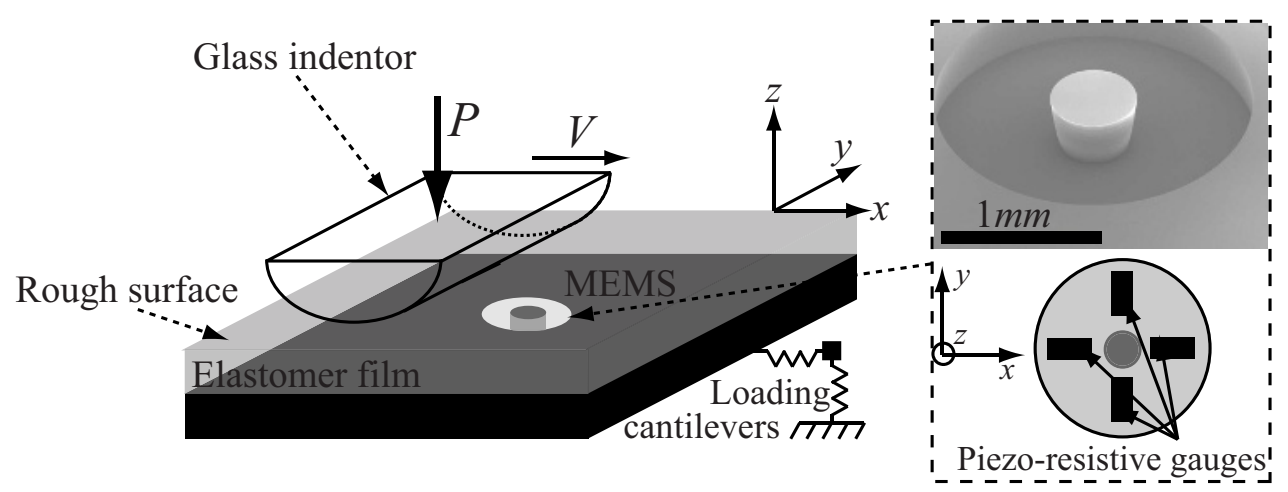

Fig. 1. Adapted from [10]. Sketch of the experimental setup. A cylindrical glass indentor (alternatively spherical) is put into contact against a rough, nominally flat PDMS elastomer film at a constant prescribed normal load $P$ and a constant velocity $V$. The local normal and tangential stress at the rigid base of the film are measured by a MEMS force sensor (lower inset). $P$ and the tangential load $Q$ applied on the film are measured through the extension of two orthogonal loading cantilevers. 
The stress sensing device (MEMS with its PDMS film) has been calibrated in [8,10], with a procedure that is only summarized here. The surface of the film is indented with a rigid cylindrical rod of diameter $500 \mu \mathrm{m}$, under a normal load $P$. With this flat punch indentor, all sensor outputs are found to be linear with $P$. By successively varying the position of this rod along the $x$ direction, and assuming homogeneity of the surface properties of the film, the radial profiles of the normal and tangential output voltages, respectively $U_{z z}(x)$ and $U_{x z}(x)$, are constructed point by point. These profiles are then compared to the results of finite elements (FEM) calculations (Software Castem 2007) for the stress $\sigma_{z z}$ and $\sigma_{x z}$ at the base of a smooth axi-symmetrical elastic film (with the same elastic moduli and thickness as in the experiment) perfectly adhering to its rigid base and submitted to a prescribed normal displacement over a central circular area of diameter $500 \mu \mathrm{m}$. We then relate the measured output voltage $U$ to the stress field at the base of the film $\sigma$ by $U_{\alpha z}(x, y)=A_{\alpha z} G_{\alpha z} \otimes \sigma_{\alpha z}(x, y)$, where $\alpha=x$ or $z$. $A_{z z}$ and $A_{x z}$ are conversion constants (units of $\left.m V / P a\right), G_{z z}$ and $G_{x z}$ are normalized apparatus functions and $\otimes$ is a convolution product. $G_{z z}$ and $G_{x z}$ are deconvoluted in Fourier space. They both exhibit a bell shape with a typical width of the order of $600 \mu \mathrm{m}$ [10], comparable to the lateral dimension of the sensitive part of the MEMS. Note that we use the sign convention that $\sigma_{z z}$ is positive for compressive loading.

\section{Axisymmetric static contact under normal load}

Static sphere-on-plane MCIs are formed, under various normal loads $P$ in the range $0.34-2.75 \mathrm{~N}$, against an optical plano-convex spherical glass lens (radius of curvature $128.8 \mathrm{~mm}$ ) [8]. Both the glass and PDMS surfaces are passivated using a vapor-phase silanization procedure which lowers and homogenizes the surface energy. As for the rod indentation, the radial profiles are derived from a series of 33 contacts whose centers lie every $0.5 \mathrm{~mm}$ along the $x$ direction. These profiles divided by $A_{z z}$ have the dimension of a stress and are labelled $S_{z z}$.

For the sake of quantitative comparison, we performed finite element calculations for a frictional sphere-on-plane contact with the same geometry as in the experiments. Both solids are smooth and the interface locally obeys Amontons' friction law with an adjustable friction coefficient $\mu$. We use a uniform mesh size of $50 \mu \mathrm{m}$ and we impose the normal displacement of the rigid sphere. The contact is solved using a double Lagrange multiplier (both surfaces are slave and master). We increase step-bystep the normal load and at each step an iterative Newton-Raphson method is used to satisfy both the

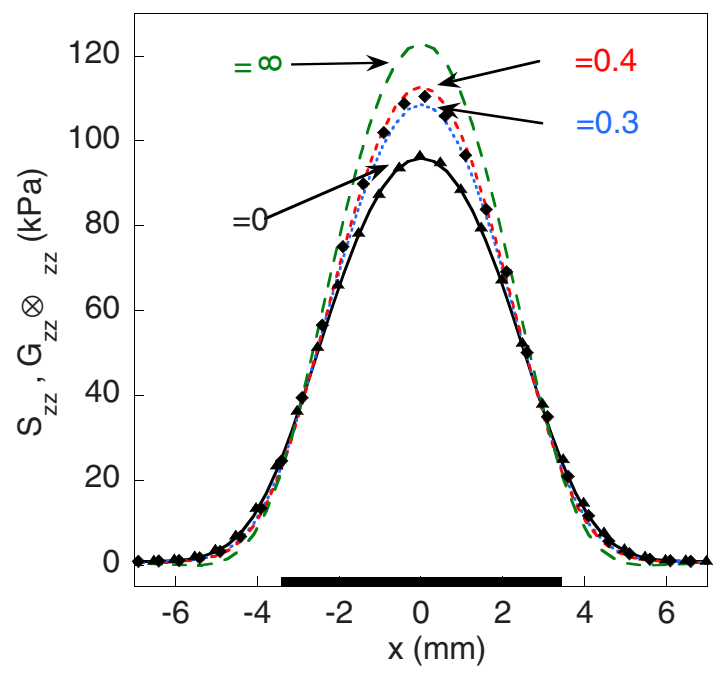

Fig. 2. Adapted from [8]. Measured normal stress profiles $\left(S_{z z}\right)$ for $P=2.75 \mathrm{~N}$, for both dry $(\bullet)$ and glycerollubricated ( - ) contacts. They are compared with the $G_{z z} \otimes \sigma_{z z}$ profiles for 4 values of $\mu(0,0.3,0.4, \infty$, shown in solid and dashed lines). The bottom patch represents the contact diameter $(3.45 \mathrm{~mm})$ in the calculations for $\mu=0$. 
unilateral contact and the friction law. In particular, we compute the radial normal stress profile $\sigma_{z z}(x)$ at the base of the film.

On figure 2 we show, on the example of a normal load $P=2.75 \mathrm{~N}$, the $S_{z z}$ profiles for both dry and lubricated conditions (glycerol droplet inserted at the interface prior to loading), together with the $G_{z z} \otimes \sigma_{z z}$ profiles for various coefficients of friction between $\mu=0$ and $\mu=\infty$. The lubricated profile is correctly captured by the finite element calculations for $\mu=0$. The dry profile is satisfactorily approached using an effective friction coefficient $\mu_{e}$ which can be determined by trial and error with a resolution of order 0.1 (see fig. 2 where $\mu_{e}$ lies between 0.3 and 0.4 ). These two observations are true for all loads higher than $\simeq 1 \mathrm{~N}$. At lower loads, the lubricated profiles cannot be approximated by any of the calculated profiles (see discussion below).

In order to show the load dependence of $\mu_{e}$, we plot the maximum measured pressure $S_{\text {max }}$ normalized by the maximum calculated pressure $p_{\max }^{0}$ for $\mu=0$, for different loads. We compare this evolution with Amontons' behaviour by overplotting the equivalent calculated quantity $p_{\max }^{\mu} / p_{\max }^{0}$ for different friction coefficients $\mu$ in the range $[0, \infty]$. One can then read off fig. 3 and evaluate the effective friction coefficient $\mu_{e}$ for any load. $\mu_{e}$ is an increasing function of $P$ and assumes values much lower than $\mu_{\text {macro }} \approx 1.6$, the macroscopic friction coefficient (measured independently for a driving velocity $v=100 \mu \mathrm{m} / \mathrm{s})$.

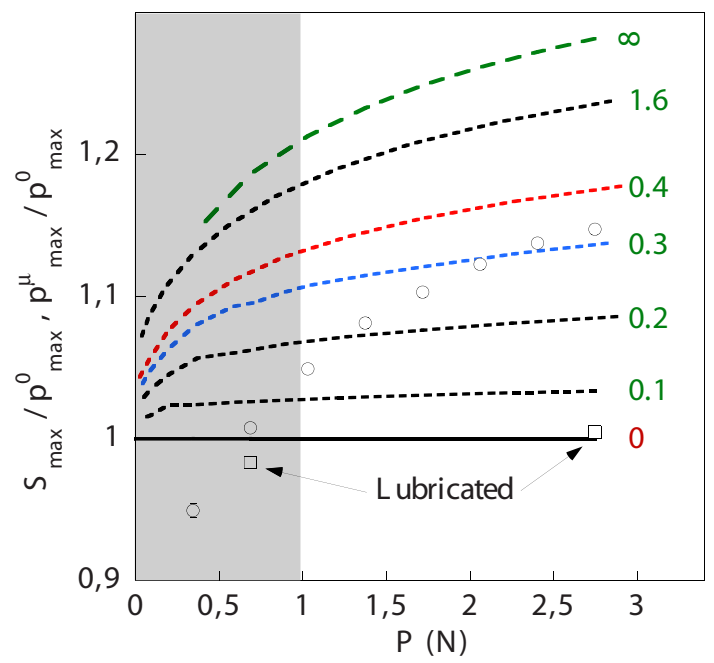

Fig. 3. [8]. Load dependence of $S_{\max } / p_{\max }^{0}$ for dry $(\bigcirc)$ and lubricated ( $\square$, guideline in dashed line) contacts, and $p_{\max }^{\mu} / p_{\max }^{0}$ for various values of $\mu$. Outside of the grey region, the normal compliance effect is negligible.

The incompatibility of the measurements with Amontons' law suggests that the latter rigid-plastic behaviour law should be refined. A dominant role of adhesion can be ruled out since, in contradiction with fig.3, it would induce a decreasing friction coefficient with normal load [40]. We are therefore led to question the rigid hypothesis. As a matter of fact, MCI's are known to possess finite compliances both in normal and in-plane directions. The effect of the normal compressibility of a rough interface $[30,35,41]$ on a sphere-on-plane contact has first been described by Greenwood and Tripp [42]. The apparent contact radius is larger than for a smooth interface and the maximum normal stress is smaller. The higher the ratio of the indentation depth to the $r m s$ surface roughness $\rho$, the larger the effect. The tangential compliance of MCI has been recently probed experimentally [43-45]: the interface deforms reversibly before irreversible slippage when the shear deformation reaches values of the order of $\rho$.

Here, the effect of the two compliances can be uncoupled by comparing dry and lubricated contacts for the same load. Lubrication suppresses the tangential stress, and the only remaining effect comes from the MCI normal compressibility. In this respect, Fig.3 shows that the latter is negligible over 
$P \gtrsim 1 N$ (outside the grey region). However, it is probably responsible for the fact that $S_{\max } / p_{\max }^{0}$ falls below 1 for the smallest loads. The tangential compliance of the interface is thus expected to be primarily responsible for the observed drop from $\mu_{\text {macro }}$ to $\mu_{e}$ for dry contacts. It can be understood qualitatively in the following way: imagine a sphere-on plane MCI with finite friction for which, temporarily, the asperities cannot deform tangentially. A slip annulus at the periphery of the contact region coexists with a central circular stuck region [23]. If we now allow the asperities to deform, they will allow for a partial strain relaxation which is qualitatively equivalent to reducing the apparent friction coefficient. This effect should vanish at higher loads, as the slip becomes larger than the maximum tangential relaxation $\simeq \rho$ allowed by the MCI deformation. The effective friction coefficient $\mu_{e}$ is therefore expected to asymptotically reach $\mu_{\text {macro }}$ as the load $P$ is increased, in qualitative agreement with our observations (fig.3).

\section{Bidimensional steady sliding frictional contact}

Steady sliding MCIs are formed, under various normal loads $P$ in the range $0.34-2.75 \mathrm{~N}$, against an optically smooth plano-convex cylindrical glass lens (radius of curvature $129.2 \mathrm{~mm}$ ), driven over $20 \mathrm{~mm}$ along the positive $x$ direction at constant velocity $V=1 \mathrm{~mm}^{-1}$ [10]. We derive the stress profiles along the sliding direction directly from the time dependence of the MEMS signals through the relation $S_{\alpha z}(x)=U_{\alpha z}(V t) / A_{\alpha z}$ (with $\alpha=x, y$ and $z$ ). We checked that the $x$ profiles are reasonably $y$-invariant over a few millimeters, thus providing an experimental realization of a 2-dimensional cylinder-onplane friction experiment.

For the sake of direct quantitative comparison, we have developed the following original bidimensional quasi-static model [10]: A linear incompressible elastic film, of thickness $h$ and Young's modulus $E$, is loaded under plane strain conditions by a rigid circular body of radius $R$ moving at a constant velocity $V$. The boundary conditions are (i) a perfect adhesion of the film to its rigid base (ii) Amontons' law of friction within the contact with a dynamic friction coefficient $\mu_{d}$ (iii) no traction outside the contact and (iv) a prescribed normal displacement induced by the circular indentor over the contact zone of width $2 a$. The input parameters of the model are $\mu_{d}$ and $P_{L}$ the lineic normal load applied. We compute both the normal and tangential stress $x$-profiles at the base of the film. We emphasize that this model is exact in the sense that, contrary to most previous works (see e.g. [46-50], $[51,52]$ being among the exceptions), it takes full account of the normal tangential coupling at the contacting interface.

We now compare directly the measured stress profiles $S_{z z}(x)$ and $S_{x z}(x)$ with the stress profiles $\sigma_{z z}(x)$ and $\sigma_{x z}(x)$ calculated at the base of the elastic film and convoluted with the apparatus functions $G_{z z}$ and $G_{x z}$ (Fig. 4). The input parameters $P_{L}$ and $\mu_{d}$ are extracted from the measured stress profiles as $P_{L}=\int_{-\infty}^{\infty} S_{z z} d x$ and $\mu_{d}=\int_{-\infty}^{\infty} S_{x z} d x / \int_{-\infty}^{\infty} S_{z z} d x$. With such definitions, $P_{L}$ is found to increase from 20 to $220 N . \mathrm{m}^{-1}$ and $\mu_{d}$ to decrease from 2.6 to 2.0 when $P$ varies from 0.34 to $2.75 \mathrm{~N}$. These values are to be compared to the macroscopic friction coefficient $Q / P$, which is a decreasing function of $P$ (and thus of the local pressure), assuming values from $1.5 \pm 0.1$ at $P=0.34 \mathrm{~N}$ down to $1.36 \pm 0.04$ at $P=2.75 \mathrm{~N}$. The latter are typical values for PDMS on glass steady sliding contacts (see e.g. $[53,54]$ ).

Figure 4 shows that the calculation provides a reasonable agreement with the experimental profiles. In particular, they account for both the negative part of $S_{z z}(x)$ and the dip of $S_{x z}(x)$ at negative $x$. In order to quantify the deviations between the experimental and calculated profiles, we compute the quantity $\chi=\sqrt{\Sigma_{i}\left(E_{i}-C_{i}\right)^{2} / \Sigma_{i} E_{i}^{2}}$, where $E_{i}$ are the experimental data points and $C_{i}$ are the calculated ones. For the tangential stress $\chi=11 \pm 1 \%$, for the normal stress, $\chi=11 \pm 3 \%$, with no clear load dependance over the range of $P$ explored. We checked that, in the present experimental conditions, the exact model provides a clear improvement with respect to previous approximate models, especially as the ratio of contact size $a$ to film thickness $h$ becomes large.

However, robust non-negligible deviations are observed, the origin of which is not elucidated yet. Two central assumptions used in the model are in particular amenable to refinement and can be considered to explain the observed deviations. First, the interface is assumed to be molecularly smooth whereas the surface of the elastomer exhibits a micrometric roughness. As discussed in section 3 , the 

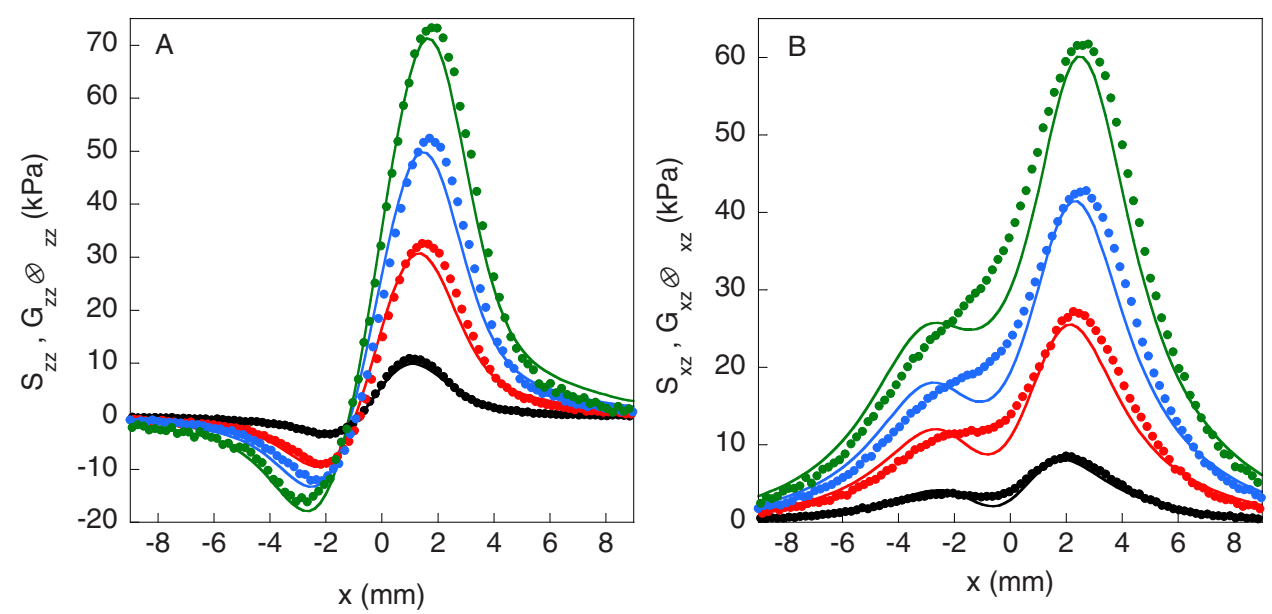

Fig. 4. Adapted from [10]. Comparison between the measured stress profiles (discs) and the model predictions (lines) for increasing normal loads $(P=0.34 \mathrm{~N}$ in black, $1.03 \mathrm{~N}$ in red, $1.72 \mathrm{~N}$ in blue and $2.75 \mathrm{~N}$ in green) and $V=1 \mathrm{~mm} \cdot \mathrm{s}^{-1}$. A: $S_{z z}$ and $G_{z z} \otimes \sigma_{z z}$. B: $S_{x z}$ and $G_{x z} \otimes \sigma_{x z}$.

resulting MCI is thus expected to exhibit finite compressive and shear compliances. This feature is known to modify, with respect to smooth contacts, both the stress $([42,8,9])$ and displacement ([45]) fields. However, these effects are expected to induce vanishing corrections at increasingly high loads. The absence of observed load-dependence of the deviations suggests that the roughness effect may not be the primary cause for deviations. The second questionable assumption is the existence of a single pressure-independent friction coefficient. This is clearly at odds with the observed decrease of $Q / P$ as a function of $P$, a behavior that is usually attributed to the finite adhesion energy of the interface (e.g. [40]). In order to take this effect into account in the model, one should let the local friction coefficient depend on the location within the contact region, according to the value of the contact pressure. Such an improvement is left for future work.

\section{Conclusion}

We have described a MEMS-based set-up allowing for spatially resolved measurements of both the normal and tangential stress fields at the rigid base of a compliant layer. On the practically relevant situation of elastic rough surfaces bearing micrometer-sized asperities, we considered two classical types of loading in contact mechanics: (i) the static normal indentation by a rigid sphere and (ii) the steady sliding indentation by a rigid cylinder. The measurement technique used here is of very broad interest in contact mechanics because they provide a significantly richer information than the classical measurements of the total normal and shear forces $P$ and $Q$. We emphasize that they allow for measurements in any loading regime, either static or dynamic, and could be of great help in other domains such as rheology or adhesion, where accurate spatially resolved stress measurements at interfaces are also needed.

The other important aspect is that the measurement are accurate enough to test quantitatively any behaviour law at the interface. In the present work, we demontrated this possibility on the example of Amontons' law of friction, which is believed to broadly apply for macroscopic multicontacts. We showed that Amontons' law fails to describe static frictional MCIs whereas it fits satisfactorily the steady-sliding frictional MCIs. This suggests that more generally, Amontons' law will fail as soon as the contact region is divided into distinct sub-regions where stick zones are adjacent to slip zones. This is not the case for instance for the lubricated static contacts or for the steady-sliding contacts, in which the whole contact is a slip zone, and where Amontons' law was indeed successful. We believe that this 
behaviour is a consequence of the finite tangential compliance of MCIs and that the related effects on the contact stress have up to now received too little attention.

\section{References}

1. K.L. Johnson, Contact Mechanics (Cambridge university Press, 1985)

2. D. Hills, D. Nowell, Mechanics of Fretting Fatigue (Kluwer Academic Publishers, 1994)

3. K. Holmberg, A. Laukkanen, H. Ronkainen, K. Wallin, S. Varjus, J. Koskinen, Surface and Coatings Technology 200(12-13), (2006) 3793

4. M. Guagliano, M. Pau, Tribology International 40(7), (2007) 1147

5. B. Luan, M. Robbins, Tribology Letters 36, (2009) 1

6. K. Kato, Wear 241(2), (2000) 151

7. T. Baumberger, C. Caroli, Advances in Physics 55(3), (2006) 279

8. J. Scheibert, A. Prevost, J. Frelat, P. Rey, G. Debrégeas, EPL 83(3), (2008) 34003

9. A. Chateauminois, C. Fretigny, European Physical Journal E 27, (2008) 221

10. J. Scheibert, A. Prevost, G. Debrégeas, E. Katzav, M. Adda-Bedia, Journal of the Mechanics and Physics of Solids 57(12), (2009) 1921

11. S. Cheng, B. Luan, M. Robbins, Physical review E 81, (2010) 016102

12. Y. Mo, I. Szlufarska, Physical Review B 81, (2010) 035405

13. R. Howe, M. Cutkosky, IEEE Transactions on Robotics and Automation 9, (1993) 140

14. L.A. Jones, S.J. Lederman, Human Hand Function (Oxford University Press, New York, 2006)

15. J. Scheibert, S. Leurent, A. Prevost, G. Debrégeas, Science 323, (2009) 1503

16. A. Prevost, J. Scheibert, G. Debrégeas, Communicative and Integrative Biology 2, (2009) 422

17. J. Scheibert, A. Prevost, G. Debrégeas, A MEMS-based tactile sensor to study human digital touch: mechanical transduction of the tactile information and role of fingerprints, in International Conference of Experimental Mechanics 14, Poitiers, France, article 473 (2010)

18. C. Scholtz, The Mechanics of Earthquakes and Faulting (Cambridge University Press, 1990)

19. S. Di Carli, C. Voisin, F. Cotton, F. Semmane, Journal of Geophysical Research-Solid Earth 113(B5), (2008) B05307

20. B.N.J. Persson, I.M. Sivebaek, V.N. Samoilov, K. Zhao, A.I. Volokitin, Z. Zhang, Journal of Physics-Condensed Matter 20(39), (2008) 395006

21. C. Cattaneo, Rendiconti dell'Accademia nazionale dei Lincei 27, (1938) 214

22. R.D. Mindlin, Trans. ASME, Series E, Journal of Applied Mechanics 16, (1949) 259

23. D.A. Spence, J. Elasticity 5(3), (1975) 297

24. G.M. Hamilton, Proceedings of the Institution of Mechanical Engineers C: Journal of Mechanical Engineering Science 197(1983), (1983) 53

25. A. Brzoza, V. Pauk, Journal of Theoretical and Applied Mechanics 45(2), (2007) 277

26. A. Brzoza, V. Pauk, Archive of Applied Mechanics 78(7), (2008) 531

27. K. Vorvolakos, M. Chaudhury, Langmuir 19(17), (2003) 6778

28. L. Bureau, T. Baumberger, C. Caroli, Eur. Phys. J. E 19(2), (2006) 163

29. F. Bowden, D. Tabor, The Friction and Lubrication of Solids (Oxford University Press, 1950)

30. J.A. Greenwood, J.B.P. Williamson, Proc. R. Soc. Lond. A 295(1442), (1966) 300

31. S. Brown, C. Scholz, J. Geophys. Res. 90, (1985) 5531

32. B.N.J. Persson, J. Chem. Phys. 115(8), (2001) 3840

33. K.A. Nuri, Wear 30(3), (1974) 321

34. J. Kagami, K. Yamada, T. Hatazawa, Wear 87(1), (1983) 93

35. M. Benz, K. Rosenberg, E. Kramer, J. Israelachvili, J. Phys. Chem. B 110(24), (2006) 11884

36. B. Lorenz, B.N.J. Persson, Journal of Physics-Condensed Matter 21(1), (2009) 015003

37. B.J. Kane, M.R. Cutkosky, G.T. Kovacs, Sens. Actuators A: Phys. 54(1-3), (1996) 511

38. M. Leineweber, G. Pelz, M. Schmidt, H. Kappert, G. Zimmer, Sens. Actuators A: Phys. 84(3), (2000) 236

39. C.M. Oddo, L. Beccai, M. Felder, F. Giovacchini, M.C. Carrozza, Sensors 9(5), (2009) 3161

40. G. Carbone, L. Mangialardi, Journal of the Mechanics and Physics of Solids 52(6), (2004) 1267 
41. B.N.J. Persson, Phys. Rev. Lett. 99(12), (2007) 125502

42. J.A. Greenwood, J.H. Tripp, Trans. ASME E, J. Appl. Mech. 34, (1967) 153

43. P. Berthoud, T. Baumberger, Proc. R. Soc. A 454(1974), (1998) 1615

44. L. Bureau, C. Caroli, T. Baumberger, Proc. R. Soc. Lond. A 459, (2003) 2787

45. J. Scheibert, G. Debrégeas, A. Prevost, Arxiv:0809.3188v1 (2008)

46. R.B. King, T.C. O’Sullivan, International Journal of Solids and Structures 23(5), (1987) 581

47. D. Nowell, D.A. Hills, International Journal Of Solids And Structures 24(1), (1988) 105

48. S.A.G. Oliveira, A.F. Bower, Wear 198(1-2), (1996) 15

49. A.A. Elsharkawy, International Journal of Solids and Structures 36(26), (1999) 3903

50. Z. Shi, S. Ramalingam, Surface and Coatings Technology 138(2-3), (2001) 192

51. L.F. Ma, A.M. Korsunsky, International Journal of Solids and Structures 41(11-12), (2004) 2837

52. L.F. Ma, A.M. Korsunsky, K. Sun, Journal of Tribology - Transactions of the ASME 128(4), (2006) 886

53. A. Galliano, S. Bistac, J. Schultz, Journal of Colloid and Interface Science 265(2), (2003) 372

54. F. Wu-Bavouzet, J. Clain-Burckbuchler, A. Buguin, P.G. De Gennes, F. Brochard-Wyart, The Journal of Adhesion 83(8), (2007) 761 